\title{
Les tiers, au cour de la dynamique conflictuelle
}

Pascal Hintermeyer ${ }^{1}$

Université de Strasbourg

UMR 7367 Dynamiques européennes

Paru dans Négociations, 2015, n² 2, p. 131-142

Résumé :

La dynamique conflictuelle implique souvent plusieurs tiers. En analysant leurs rôles, l'auteur montre qu'ils s'étendent et se diversifient. Ainsi les observateurs, les conciliateurs, les experts ont pris de l'importance. Ces évolutions conduisent à une objectivation et à une rationalisation, mais aussi à une prise en compte des aspects émotionnels et de la souffrance issue des conflits.

Mots-clés: Tiers - dynamique conflictuelle - observateurs - médiateurs experts -souffrance

Summary :

The conflicting dynamics often involves several thirds. Analysing the roles that they play, the author shows that they are expanding and diversifying. So the observers, the conciliators and the experts become more important. These evolutions lead to more objectification and rationalization, but also to a consideration of the emotional aspects and of the suffering due to the conflicts.

Key words : Third - conflicting dynamics - observers - mediators - experts suffering

Depuis Clausewitz, la guerre est analysée comme la conséquence de l'affrontement entre deux volontés opposées dans un processus de montée aux extrêmes. La polarisation duelle s'envenime dans des situations où aucun tiers n'est en mesure de s'interposer et d'imposer le respect mutuel. Plus généralement, les relations sociales peuvent être envisagées à partir de discordes susceptibles de dégénérer. Déjà chez Hobbes, les volontés discordantes provoquent une animosité généralisée menaçant l'existence de chacun jusqu'à ce que ce tiers qu'est l'Etat oblige chacun à renoncer à ses exigences, rendant possible la concorde et l'ordre social. Plus récemment, Julien Freund définit « le conflit comme la relation sociale marquée par le tiers exclu. » (Freund, 1983 : 287) Il montre qu'au contraire, la réintroduction du tiers peut orienter vers l'acceptation de règles communes et vers le compromis. Il prolonge ainsi l'analyse de Georg Simmel, qui explique que le tiers atténue les passions

\footnotetext{
${ }^{1}$ Pascal.Hintermeyer@misha.fr
} 
conflictuelles en amenant les adversaires à temporiser et en déplaçant le conflit $\mathrm{du}$ registre affectif au registre intellectuel. L'affrontement des subjectivités cède alors devant la prise en compte des intérêts objectifs. Le tiers impartial peut ainsi désamorcer les aspects affectifs de la rivalité et orienter la dynamique conflictuelle vers la négociation. Le tiers peut d'ailleurs agir efficacement même lorsque son intervention reste potentielle ou lorsqu'il ne se présente que sous la forme impersonnelle de normes s'imposant aux deux parties. De ces analyses, il ressort que le tiers est un moyen pour réguler la conflictualité, la maintenir dans des limites socialement acceptables ou tirer parti de son potentiel créateur. Mais il convient également de souligner que le tiers peut avoir d'avoir des effets différents, voire contraires. En suivant Simmel qui a aussi envisagé cette possibilité et en commentant les explications qu'il a données à ce sujet, nous montrerons l'intérêt d'une réflexion visant à compléter la typologie qu'il a élaborée et qui a été fréquemment reprise ultérieurement. Cela nous conduira à attirer l'attention sur l'extension et la diversification du rôle du tiers ainsi que sur des situations caractérisées par la pluralité ou la multiplicité des tiers. Les avantages liés à la position de tiers incitent en effet à une concurrence entre eux. Dans le mouvement contemporain de prolifération des tiers, nous relèverons l'importance croissante prise par la médiation, la souffrance et l'expertise ainsi que par les tentatives visant à conjuguer ces deux foyers de légitimation.

\section{L'implication des tiers dans les conflits}

L'influence du tiers n'a rien d'univoque ou de systématique. En contrepoint des situations où elle s'avère pacificatrice, Simmel envisage aussi des cas où le tiers peut envenimer un conflit. Cela peut paradoxalement se produire lorsqu'il bénéficie d'une révérence incontestée, par exemple lorsqu'il est invoqué comme la science ou la vérité : «L'opposition qui s'est ainsi constituée entre l'unité et l'antagonisme est peut-être la plus sensible là où les deux parties poursuivent véritablement un seul et même but, par exemple pour établir une vérité scientifique. » (Simmel, 1992: 54) Une référence que les adversaires sont enclins à placer au-dessus de leurs propres intérêts, qui semblerait donc les faire converger vers une valeur susceptible de procurer l'apaisement d'ordinaire attendu du tiers, peut au contraire radicaliser leur antagonisme. Et, bien sûr, le tiers peut jouer un rôle polémogène lorsqu'il suscite un conflit ou en profite.

Simmel distingue ici deux possibilités : «à l'intérieur de cette forme, on trouvera principalement deux réalisations : deux parties sont ennemies et par conséquent rivalisent pour obtenir la faveur d'un tiers; ou bien : deux parties rivalisent pour obtenir la faveur d'un tiers et sont par conséquent ennemies. » (Simmel, 1999 : 140) Dans ces deux situations, le tiers se trouve absorbé par le conflit. Dans la première, il est recherché comme allié potentiel par les protagonistes d'un affrontement déjà en place. Dans la seconde, c'est le tiers luimême qui est l'origine et la cause du conflit. Simmel remarque que, dans le 
premier cas, le ralliement du tiers marque le début du combat et il ajoute : «A l'inverse, quand deux éléments cherchent indépendamment l'un de l'autre à obtenir la faveur d'un tiers, et que c'est là la raison de leur animosité, de leur division en deux parties, l'attribution finale de cette faveur -qui est donc l'objet et non le moyen du combat- y mettra fin. » (ibid.) Simmel pense par exemple à la rivalité entre deux prétendants pour la conquête d'une femme mais on peut estimer, même dans ce cas, sa conclusion exagérément optimiste. Il apparaît en tout cas que chez Simmel, le tiers peut jouer un rôle très variable : il peut prévenir, réguler et atténuer le conflit, mais il peut aussi stimuler le conflit, en s'y mêlant, en le provoquant ou en l'entretenant. Et il peut faire les deux à la fois, surtout lorsqu'il recouvre une pluralité de positions. Avant de mettre en relief cette ambiguïté, nous pouvons noter dès à présent que le tiers, soit qu'il attise le conflit, soit qu'il l'apaise ou le transforme, est au cœur de la dynamique conflictuelle.

Simmel développe un exemple de situation où le tiers est la raison du conflit, c'est la concurrence, qu'il oppose à des formes plus primitives de confrontation. "Se battre avec quelqu'un, c'était combattre pour s'emparer de lui et le réduire en esclavage; au lieu de cela, on a donc ce phénomène complexe qu'est la concurrence, dans laquelle un homme se bat contre un autre, certes, mais pour en séduire un troisième.» (Simmel, 1992: 82) Ce troisième homme à séduire, que Simmel présente par ailleurs comme «l'exemple le plus pur de tertius gaudens » (Simmel, 1999: 141), ce larron qui se réjouit de la rivalité entre les deux autres parce qu'il espère en tirer des opportunités de profit pour lui-même, n'est-il pas plutôt à envisager comme un ensemble disparate composé d'un grand nombre de personnes qui n'agissent nullement de manière unifiée ou coordonnée ? Ce que Simmel appelle « la clientèle» ((ibid.) est en fait une population, dont on peut penser que tous ceux qui en font virtuellement partie sont susceptibles de réagir aux sollicitations de manières divergentes. Cette diversité peut d'ailleurs être considérée comme la condition même du maintien de la concurrence, une forme d'action réciproque qui ne subsiste que parce que tous les tiers susceptibles d'être impliqués dans la relation commerciale ne prennent pas tous la même décision, ce qui se traduirait par la ruine du concurrent unanimement délaissé. L'analyse de Simmel montre d'ailleurs qu'à cette multitude de possibles clients s'ajoute un autre type de tiers, tout aussi essentiel à la persistance de la concurrence, mais intervenant à un autre niveau et d'une manière différente : "la limitation des moyens de la concurrence sans empêcher la poursuite de celle-ci se fait grâce à des instances situées bien au-delà des concurrents et de leurs intérêts : le droit et la morale » (Simmel, 1992: 101) On sait que ces deux instances impersonnelles ne convergent pas toujours, voire comportent chacune des tendances diverses. L'examen précis de la situation concurrentielle analysée par Simmel fait ainsi apparaître une pluralité de tiers intervenant effectivement ou potentiellement dans le conflit. La portée de cette observation s'accroît encore lorsqu'on réalise, 
à la suite de Simmel, que la concurrence ne concerne pas seulement le commerçant, mais aussi « le journaliste, l'artiste, le libraire, le parlementaire. » (id. : 79) On pourrait compléter la liste et relever que, dans bien des professions et des activités effectuées dans le cadre de sociétés différenciées, des polarisations conflictuelles se développent en impliquant de nombreux tiers.

\section{Extension du rôle des tiers}

On sait que Simmel distingue 4 types de tiers. Deux d'entre eux sont réputés limiter ou résoudre les conflits, il s'agit de l'arbitre, censé ramener impartialement les adversaires au respect des règles, et du médiateur, qui a pour tâche de rapprocher leurs points de vue. Deux autres types jouent un rôle inverse, ce sont le tertius gaudens, qui profite des opportunités offertes par un conflit et le diviseur, qui attise intentionnellement les conflits pour asseoir sa prééminence. Cette typologie, qui a été souvent reprise, et qui conserve sa pertinence, a donné lieu à quelques tentatives visant à la compléter. Ainsi, Caplow a proposé de considérer toute relation sociale comme potentiellement conflictuelle et marquée par l'influence d'un tiers que chacun des deux autres cherche à se concilier en nouant une coalition qui lui soit favorable. Il a cherché à modéliser ces coalitions en fonction de la puissance respective des trois acteurs et des alliances nouées entre eux (Caplow, 1956: 491) Il considère que la perspective tierce est impliquée dans toute relation sociale : «L'interaction est le processus social de base grâce auquel les individus et les groupes exercent une influence les uns sur les autres, et c'est un processus triangulaire - ou triadique parce qu'il subit toujours l'influence d'un public, présent ou proche.» (Caplow, 1971 : 10) Cette remarque attire l'attention sur l'idée qu'aucune relation et aucun conflit ne peut se restreindre à ses protagonistes initiaux et ne se déroule entre eux seuls, indépendamment de leur environnement.

Un conflit se situe dans un contexte qui lui donne au moins une partie de son sens, il se déploie devant des témoins qui ne s'avèrent pas seulement passifs. Depuis l'Antiquité, les conflits sont relatés par des épopées, des drames et des choeurs les évoquant lors de représentations collectives. D'autres tiers ont émergé sous les traits de chroniqueurs, mémorialistes, archivistes, historiens, ils ont ensuite été parfois relayés par des romanciers, scénaristes, cinéastes. Toutes ces voix sélectionnent les péripéties significatives d'un conflit à l'intention de la collectivité ou de la postérité. Elles concourent à la formation de l'opinion dont l'importance, à la suite de Tocqueville, a été souvent soulignée dans les sociétés démocratiques. Cette force s'implique de manière très variable dans les conflits, tantôt en les stimulant, tantôt en les évitant ou en les ignorant. La grande guerre a ainsi éclaté à la faveur d'une opinion qui s'est révélée consentante, voire enthousiaste. Elle a été suivie par une période où le pacifisme a prévalu dans certains pays. Cette sensibilité de l'opinion n'a pas empêché les conflits de s'envenimer, mais elle a induit chez les parties prenantes des postures 
spécifiques à cette époque. Ce tiers qu'est l'opinion s'avère donc à la fois changeant et contraignant dans les dynamiques conflictuelles observables.

Comme la clientèle, l'opinion apparaît généralement divisée. Ce qu'elle comporte d'instable, d'impérieux et de déroutant explique le soin mis à étudier ses tendances, ses variations, ses hésitations. (Stoetzel, 1943) Cette complexité suscite des tentatives pour l'exprimer, l'infléchir ou la manipuler. Elle permet aussi à divers tiers de s'immiscer dans des conflits en l'invoquant. C'est en particulier à travers leurs rapports étroits à l'opinion que les médias font figure de $4^{\mathrm{e}}$ pouvoir. Or ceux-ci consacrent une grande part de leurs ressources à rendre compte des conflits. Ils interviennent par là même dans les controverses animant le débat public. Leur attitude active se manifeste aussi à ce qu'ils missionnent des reporters et autres envoyés spéciaux pour "couvrir» les conflits, en obtenant des garanties pour leur protection. Ils sont parvenus à faire reconnaître leur statut de tiers par cet autre tiers que constitue l'Organisation des Nations Unies. En effet, le Conseil de sécurité de cette instance internationale a adopté à l'unanimité le 23 décembre 2006 la résolution 1738 sur la protection des journalistes dans les conflits armés. A la différence des situations où la pluralité des tiers aboutit à une rivalité entre eux, il arrive ainsi qu'ils s'organisent pour se renforcer mutuellement.

Même lorsque leur travail d'investigation reste limité, les activités des médias ne se bornent pas à la transcription de combats existant préalablement. Elles influent sur l'événement, voire contribuent à le produire. En prenant l'exemple du hooliganisme au stade du Heyssel, Baudrillard va jusqu'à soutenir que c'est le dispositif mis en place en de tels lieux qui stimule l'attente des affrontements destinés à s'y produire. (Baudrillard, 1990) L'amorce de ce genre de processus peut apparaître dans une cour d'école : un cercle se forme pour profiter de l'opportunité offerte par une bagarre, en protéger la possibilité, délimiter l'espace où elle se déroulera, stimuler les combattants. Les observateurs ne sont pas sans effet sur le conflit auquel ils assistent. Ils peuvent contribuer à l'attiser et à l'entretenir, même lorsqu'ils n'en espèrent pas d'avantage personnel. Ils peuvent aussi s'en détourner en le laissant se poursuivre, mais en portant leur attention sur un autre conflit qui présente des attraits différents, parfois simplement celui de la nouveauté. Les observateurs sont aussi devenus une ressource en vue de la stabilisation et du traitement des conflits. Ils sont notamment fréquemment envoyés dans les zones d'affrontement ainsi que lors de processus électoraux menaçant de dégénérer. La Charte des Nations Unies les mentionne dans son chapitre 6 sur le Règlement pacifique du conflit (leur déploiement devant être négocié avec les autorités des pays concernés) et au chapitre 7 (à condition qu'aucun membre du Conseil ne sécurité ne fasse usage de son droit de veto) concernant « les actions en cas de menace contre la paix, de rupture de la paix et d'acte d'agression », avec dans ce cas l'obligation pour les Etats d'autoriser leur venue et d'assurer leur sécurité. Un même type de tiers, en l'occurrence celui qui se recommande de l'exercice 
de la faculté d'observation, peut tantôt se réjouir d'un conflit, pour différentes raisons, tantôt s'en désintéresser, tantôt s'interposer. La position d'observateur illustre ainsi l'extension du rôle des tiers ainsi que la diversification de leur implication. De même, les nombreux spectateurs et commentateurs que les conflits contemporains charrient avec eux ont sur ces derniers un impact variable et instable. La position des tiers évolue parallèlement à la diversification des opinions et de leur expression.

\section{Médiateurs et conciliateurs}

L'extension du rôle du tiers apparaît aussi à travers l'évolution des fonctions de médiation et d'arbitrage. Les premières se sont multipliées dans de nombreux domaines qui impliquent généralement des accords contractuels mais où des divergences peuvent déboucher sur des conflits. Elles peuvent par exemple concerner les relations familiales, professionnelles, de voisinage, etc. Certains de ces problèmes étaient précédemment pris en charge par les juges de paix, mis en place dans chaque canton français entre 1790 et 1958 et dont se sont inspirés divers pays européens. (Toussaint, 1958) La médiation a ensuite pris le relais en se spécialisant et en se professionnalisant. Elle en est aussi venue à porter sur quantité de différends entre le citoyen et les institutions auxquelles il recourt. Des journaux, des chaines de radio et de télévision, des services et équipements ont ainsi instauré des médiateurs pour réguler leurs relations avec leurs lecteurs, auditeurs, téléspectateurs, usagers. Leurs interventions ne visent pas seulement à apaiser les conflits en prenant en compte les différents points de vue, y compris ceux qui sont rarement entendus, elles visent aussi à les prévenir, en ouvrant aux contestations un espace d'expression argumentée, et elles offrent l'opportunité d'une réflexion a posteriori sur les pratiques constatées. Ce faisant, elles révèlent d'éventuelles dérives, mettent en évidence les choix implicites et incitent à s'interroger sur des habitudes contestables.

Un type de médiation a pris une ampleur remarquable, il concerne les relations conflictuelles entre les citoyens et les administrations dont ils relèvent. Celles-ci se sont souvent dotées de médiateurs, ce qui est le cas de divers ministères (finances, intérieur, agriculture, etc.), de certaines de leurs directions (par exemple celle de la police nationale) et de leurs antennes régionales (avec par exemple un médiateur dans chaque académie du ministère de l'éducation nationale qui dispose de plus au niveau central de sa médiatrice, assistée d'un adjoint et de chargés de mission). Par ailleurs, les médiateurs sont devenus des auxiliaires dans l'exercice ordinaire de la justice. Dans certaines affaires qu'il n'estime pas trop grave, le juge peut leur confier la mission de faciliter les échanges entre les parties en conflit, non seulement au civil mais aussi au pénal. La médiation pénale vise à établir un dialogue entre l'auteur des faits poursuivis et la victime, avec la perspective de déboucher sur une réconciliation par la 
réparation des torts subis en contrepartie du retrait de la plainte. Cette visée restauratrice est ambitieuse puisqu'elle tend à dépasser le préjudice, voire à rétablir une qualité de relation dont l'altération a rendu possible la transgression de la loi. L'analyse des interactions qui se produisent dans ce cadre montre qu'elles sont effectivement susceptibles « de favoriser le décentrement cognitif des acteurs, de développer leur capacité à faire preuve d'empathie et à «accueillir » la souffrance de l'autre partie.» (Carvajal Sanchez, 2014: 86) Dans les pratiques de médiation, un tiers incite les protagonistes du conflit à examiner leur propre comportement en prenant conscience de ses effets et en particulier des souffrances qu'il a pu causer. Au-delà de la rationalité et la technicité des procédures mises en œuvre, la pratique de la médiation tend ainsi à se situer sur un registre émotionnel. Elle incite les parties à exprimer et à faire reconnaître leur souffrance. Celle-ci apparaît comme un puissant vecteur de légitimité, qui justifie souvent la prolongation de conflits invoquant la fidélité au passé, mais qui peut aussi induire à la réconciliation là où la souffrance de l'autre est entendue.

Cette valorisation de la souffrance fait évoluer la présentation et les limites de la fonction de médiateur dont l'intervention dans un conflit tend à se justifier par la défense de la partie la plus faible. Au niveau national s'institutionnalisent des missions désignées par des appellations diverses, par exemple protecteur du citoyen dans les pays francophones, défenseur du peuple dans des pays de culture hispanique. D'autres pays reprennent le terme d'ombudsman, d'origine suédoise et s'inspirent de sa fonction consistant à se faire le porte-parole des griefs de la population. En France, le changement est significatif : le médiateur de la république, créé en 1973, a été remplacé par un Défenseur des droits fondamentaux, en vertu de la loi du 29 Mars 2011. Il est chargé de défendre l'individu face aux administrations, aux établissements et organismes investis d'une mission de service public. Il peut être saisi par un enfant, quelqu'un qui se plaint de discrimination, une personne invoquant un manquement aux règles de déontologie dans le domaine de la sécurité. Le médiateur et ses collaborateurs font ainsi valoir leur sollicitude pour toute sorte de victimes.

La légitimité associée à la souffrance consécutive aux conflits incite à l'affirmation de tiers médiateurs et au dépassement des limites imparties à la médiation. Dans un monde aux interdépendances accrues, la neutralité ne se borne plus à une tentative pour rester à l'écart des conflits. Une thèse récente montre qu'elle a changé de signification et qu'elle est aussi devenue une stratégie, développée par exemple par la Suisse, qui a mené en diverses circonstances une politique active de bons offices entre belligérants en vue de la pacification. (Le Barreau, 2015) La neutralité se justifiant par l'impératif d'échapper aux souffrances générées par les conflits, elle se consolide en assumant une fonction de médiation qui, elle aussi se fait plus active, les attentes en vue de soulager la souffrance incitant à saisir toutes les opportunités qui se 
présentent, quitte à brouiller la différence avec d'autres modes d'intervention comme la conciliation.

La conciliation " consiste à charger un individu ou un groupe d'individus, représentant ou non les parties, de rechercher les causes d'un conflit, de formuler des propositions de nature à rapprocher les parties adverses et, d'une façon générale, de provoquer une détente en attendant la prise de contact directs. » (Lipstein, 1958, p. 539) Dans certains cas préalable à la médiation et à la négociation, la conciliation suggère les voies de possibles compromis. Dans les conflits du quotidien, il est souvent attendu du médiateur, dont la tâche consiste à favoriser les échanges entre les parties, qu'il assume un rôle de conciliateur, en proposant une solution au différend. (Carvajal Sanchez, ibid.) La justice a beau établir une distinction entre les deux fonctions, le conciliateur étant un bénévole qui tente de rapprocher les points de vue dans des conflits d'ordre civil, commercial ou social, le médiateur pouvant agir en tant que professionnel chargé de restaurer la communication, celle-ci tend à se focaliser sur une souffrance qui peut amener ce dernier tiers à endosser le rôle du premier.

\section{Tiers experts et tiers secourants}

D'autres tiers sont encore plus actifs et directifs dans le règlement des conflits, il s'agit du juge et de l'arbitre. Tous deux revendiquent une position d'impartialité, ils se réfèrent à des règles établies et explicites, ils prennent des décisions qui s'imposent aux parties. Ces tiers voient leur importance encore majorée dans des contextes où se multiplient les compétitions et les conflits de tous ordres. Maints exemples illustrent la judiciarisation des sociétés contemporaines, avec quantité de conflits engorgeant les tribunaux et une variété d'affaires touchant à des domaines très divers, y compris la santé, l'environnement, la consommation, etc. L'emprise de la justice s'est aussi étendue avec la création d'une justice pénale internationale en 2002. L'arbitrage est également devenu plus fréquent, notamment pour trancher des litiges financiers et commerciaux. (Fouchard, Gaillard, Goldmann, 1996) La diversité des contentieux, la technicité et la complexité de certains d'entre eux conduisent ceux qui ont la responsabilité de décider à faire appel à des experts pour être éclairés sur certains aspects impliqués par les conflits. Ce recours aux experts est une manifestation de la tendance à l'objectivation du traitement des conflits déjà relevée par Simmel. Il est aussi une conséquence de l'augmentation et de la spécialisation des connaissances mobilisables pour comprendre les tenants et les aboutissants des conflits. Ainsi s'impose un tiers légitimé par la maîtrise d'un savoir spécialisé sur le sujet du différend.

L'expert partage avec le juge et l'arbitre la revendication d'impartialité, mais il se distingue d'eux en ce qu'il n'est pas tenu de prendre en compte l'ensemble des facteurs, des contraintes et des conséquences de la décision. Il s'agit donc d'un type spécifique de tiers, chargé d'énoncer des conclusions 
précises dans un domaine de validité circonscrit. "Si le tiers est seulement chargé de fixer irrévocablement une question de fait [...], sa mission ne peut être, malgré sa force obligatoire, que celle d'un expert » (Loquin, 2009 : 537). En se bornant à constater et à établir rigoureusement des faits, l'expert pose les jalons nécessaires à la décision incombant au juge ou à l'arbitre tout en préservant son autonomie par rapport à eux. Il peut d'ailleurs aussi bien livrer ses explications aux protagonistes du conflit ou à d'autres tiers s'y impliquant. Il prend ainsi une place croissante dans les débats publics et les médias cherchant à éclairer l'opinion.

On assiste de nos jours à une prolifération d'experts et de spécialistes sollicités pour tenir un discours rationnel, circonstancié et documenté à propos de toutes sortes de conflits et de disfonctionnements. Leur crédit ne semble même pas trop affecté lorsqu'ils émettent des avis divergents ou lorsque leurs pronostics s'avèrent démentis. La position de tiers expert est donc convoitée, elle offre la perspective d'une seconde carrière à ceux dont la première a assuré la notoriété par leur participation aux compétitions et aux conflits intéressant l'opinion. L'expertise autorise ce passage du rôle de protagoniste à celui de tiers. On peut aussi relever que, tout comme la médiation, l'expertise évolue, non seulement à cause de l'augmentation des connaissances, mais aussi en raison de l'attention que l'opinion accorde à la souffrance qui, elle aussi, permet à ceux qui la prennent en charge d'échapper à la polarisation conflictuelle. «La compassion n'a pas d'ennemi. » (Fassin, 2007: 70) La souffrance résultant de situations diverses, chacune d'elle requiert une expertise articulant un savoir spécifique au traitement d'une souffrance particulière. Personnels associatifs et professionnels du travail social développent une expertise concernant les victimes de tel ou tel conflit, par exemple dans les relations familiales, l'accès au logement ou les migrations internationales. Des psychologues appliquent leurs compétences d'écoute à de multiples souffrances. Celles ayant trait au travail et aux risques psychosociaux donnent lieu à des expertises mesurant le stress et l'accroissement des exigences en milieu professionnel (Dejours, 2010). Des experts sont employés et utilisés par divers tiers, cette convergence alliant la rationalité analytique et l'émotion impliquée par la défense des victimes.

L'impératif de secourir les victimes des guerres a suscité l'émergence de nouveaux tiers, sur la base de conventions internationales, notamment celles de Genève "pour l'amélioration du sort des militaires blessés » et de La Haye, pour la protection des civils. Des initiatives se sont institutionnalisées pour porter secours aux blessés, sur le modèle de la Croix rouge créée par Henri Dunant. La volonté de porter assistance aux tiers souffrant des conséquences de conflits qui les dépassent a conduit au développement de l'action humanitaire pendant le dernier quart du XXe siècle et à la création d'organisations non gouvernementales intervenant comme tiers dans les conflits. Elles ont bénéficié de la reconnaissance internationale d'un droit d'assistance humanitaire, s'inspirant du droit d'ingérence (Bettati, 1996) et visant à permettre l'accès aux 
victimes. Elles mobilisent des experts, par exemple ceux qu' Human Rights Watch charge de répertorier les atteintes aux droits humains.

Les instances internationales tentent de s'affirmer en recourant à des experts pour mener à bien leurs missions, en particulier les opérations de maintien de la paix qui se sont multipliées depuis 1989. Cet interventionnisme a trouvé sa formulation la plus ambitieuse dans la responsabilité de protéger, doctrine élaborée par un groupe d'experts internationaux pour empêcher le renouvellement de massacres analogues à ceux de Bosnie et du Rwanda. Ce principe a été validé en 2005 par l'Assemblée générale de l'ONU et invoquée en 2011 pour justifier les interventions armées en Côte d'Ivoire et en Lybie. Ce dernier exemple illustre la puissance acquise par des instances représentant ce qu'on appelle d'habitude la " communauté internationale». Ces tiers, disposant d'experts et se légitimant par la défense des victimes, invoquent des motifs altruistes pour exercer un pouvoir décisif. Ils interviennent dans certains conflits en faisant agir d'autres tiers, pays ou alliances, dont les forces sont employées dans les affrontements.

Le rôle de l'armée de pays comme la France s'en trouve complétement modifié. Alors qu'elle était organisée pour combattre en faveur de la population et des intérêts du pays, elle guerroie de nos jours en tant que tiers se mettant au service de tiers (ONU, UE, OTAN) qui la mandatent pour investir des zones de conflits, y détruire des forces ou s'interposer entre les combattants. Elle s'y retrouve généralement en présence d'autres tiers comme des organisations régionales ou non gouvernementales. Dans cette situation de concurrence des tiers, elle se tient à distance des différents acteurs et s'efforce de s'imposer comme tiers structurant ou stratégique, contribuant à pacifier les relations au sein de la société où elle se déploie. (Ramel, 2011) D'autres armées avaient déjà tenté de passer du rôle de belligérant à celui de protecteur, par exemple celle des Etats-Unis en Allemagne et au Japon après la seconde guerre mondiale. Ces succès ont inspiré des tentatives ultérieures de construction de la paix, de reconstruction post-conflit, de Nation building et de State building. Elles ont connu bien des désillusions qui ont montré qu'il n'est pas si simple d'envahir un pays et d'y changer la société, même avec l'intention d'y apporter plus d'harmonie et de démocratie. Mais de tels exemples témoignent de l'attrait exercé par la position de tiers et aussi de la logique qui les conduit à s'engager, y compris par la force, dans certains conflits. En s'appuyant sur d'autres tiers, en particuliers experts, les décideurs et ceux qui aspirent à le devenir justifient volontiers leurs interventions en se présentant comme tiers secourants.

Conclusion

En revenant sur les conflits analysés par les auteurs qui s'y sont intéressés et en examinant ceux qui s'enveniment à différentes échelles, on se rend compte que plusieurs tiers y sont souvent impliqués et qu'ils prennent une part active 
dans leur dynamique. La diversité de ces tiers incite à compléter la typologie établie par Simmel en tenant compte de l'importance prise par des tiers comme l'opinion, les médias et les observateurs. Ces derniers peuvent agir dans des sens différents et variables, ce qui est aussi le cas de bien d'autres tiers. Ceux qui ont été précocement identifiés, comme les médiateurs, ont vu leurs attributions s'étendre, notamment aux différends entre usagers et administrations ou services, si bien qu'ils ont pu se rapprocher d'autres rôles comme ceux de conciliateur. Des tiers également identifiés de longue date, comme les arbitres, en ont généré d'autres, comme ces nombreux experts chargés d'établir des faits dans un domaine circonscrit. Ces spécialistes participent de l'objectivation des conflits, mais ils sont aussi sollicités pour pallier leurs conséquences sur les tiers qui les subissent.

La tendance à la polarisation duelle conduit généralement à faire la distinction entre les parties opposées et les tiers, mais cette différence est elle aussi instable et réversible. Certains tiers, en s'immiscant dans un conflit pour le désamorcer, peuvent être entrainés dans sa dynamique au point d'en devenir un protagoniste. Inversement, un belligérant peut tenter de se transformer en tiers en raison des avantages que procure cette position, non seulement pour poursuivre ses propres intérêts, mais aussi pour endosser le rôle de tiers secourant et bénéficier de la légitimité attachée à celui-ci. L'action des tiers ne se situe donc pas seulement sur un registre rationnel, elle fait aussi appel à des émotions associées aux souffrances issues des conflits. Cette convergence entre des dimensions généralement dissociées, entre objectivité et affectivité, entre distanciation et engagement, suscite de nombreuses vocations de tiers. Leur pluralité les conduit à entretenir entre eux des relations de concurrence ou de coopération, au cœur de la dynamique conflictuelle.

\section{Références}

BETTATI Mario (1996), Le droit d'ingérence, Paris, Odile Jacob.

BENOIST Jocelyn (2001), «Intersubjectivité et socialité : la phénoménologie et la question du tiers », in BENOIST Jean. et Bruno Karsenti (dir.),

Phénoménologie et sociologie, Paris, PUF, pp. 19-43.

BERTEN André (2005), « Du tiers au Tiers » in LEBRUN Jean-Pierre. et Elisabeth VOLCKRICK (dir.), Avons-nous encore besoin d'un tiers ?, Ramonville Saint-Agne, Erès.

CAPLOW Theodore (1971), Deux contre un. Les coalitions dans la triade, Paris, Armand Colin.

CAPLOW Theodore (1956), "A theory of Coalitions in the Triad », American Sociological Review, Vol. 21, n²4, pp. 489-493.

CARVAJAL SANCHEZ Fernando (2014), « La médiation : un processus transactionnel de régulation des conflits en droit pénal », in KLINGER Myriam 
et Sébastien SCHEHR (dir.), Les dynamiques sociales et leurs conflits : mobilisations, régulations, représentations, Chambéry, Université de Savoie, p. 77-90.

CHANET Jean-François et Christian WINDLER (dir.) (2009), Les ressources des faibles, Mayenne, Presses Universitaires de Rennes.

CHARAUDEAU Patrick.et Rosa MONTES R. (dir.) (2004), La voix cachée du Tiers. Des non-dits du discours, Paris, L'Harmattan.

DAHAN-GAIDR Laurence (dir.) (2007), Logiques du tiers. Littérature, culture, société, Presses de l'Université de Franche Comté.

DEJOURS Christophe (dir.) (2010), Observations cliniques en psychopathologie du travail, Paris, PUF.

DELANNOI GILLES, Pascal HINTERMEYER, Philippe RAYNAUD et PierreAndré TAGUIEFF (dir) (2011), Julien Freund, la dynamique des conflits, Paris, Berg international.

FASSIN Didier (2007), « Un ethos compassionnel » in SOULET M.-H. (dir), La souffrance sociale. Un nouveau malaise dans la civilisation, Fribourg, Acamedic Press Fribourg.

FREUND Julien (1975), «Le rôle du tiers dans les conflits », Etudes polémologiques, $\mathrm{n}^{\circ} 17$, pp. 11-23.

FREUND Julien (1983), Sociologie du conflit, Paris, PUF.

FOUCHARD Philippe, Emmanuel GAILLARD et Berthold GOLDMANN, (1996), Traité de l'arbitrage commercial international, Paris, Litec.

GELY Raphaël (2005), « Les Usages du tiers », in LEBRUN Jean-Pierre. et Elisabeth VOLCKRICK (dir.), Avons-nous encore besoin d'un tiers?, Ramonville Saint-Agne, Edition Erès. HOBBES Thomas (1971), Le Léviathan, Paris, Sirey (1651).

KLEINMAN Arthur, Veena DAS et Margaret LOCK (dir.) (1997), Social Suffering, University of California Press \& Oxford University Press.

LE BARREAU Lucie (2015), L'Etat tiers en relations internationales. Déclinaisons d'identités stratégiques médianes : neutralisation, finlandisation, neutralité, Thèse de doctorat en Sécurité internationale et défense, dirigée par D. Cumin, soutenue le 29 janvier 2015 à l'Université Jean Moulin Lyon III. LIPSTEIN Kurt (1958), «Introduction », in Bulletin international des sciences sociales, « Techniques de médiation et de conciliation », volume $\mathrm{X}, \mathrm{n}^{\circ} 4$, Unesco, pp. 439-440.

LITS Grégoire (2009), «Tiers et objectivité sociale chez Georg Simmel. Possibilité d'approche des sociabilités au temps de la mondialisation ?» Émulations, ${ }^{\circ} 5$, vol. 3.

LOQUIN Eric (2009), L'arbitrage, Paris Litec. 
RACINE Luc (1999), «Les formes d'action sociale réciproque: dyades et triades», Sociologie et sociétés, vol. 31, n 1 , pp. 77-92.

RAMEL Frédéric (dir .) (2011), L'UE en tant que tiers stratégique, Etudes de 1'IRSEM, ${ }^{\circ} 12$.

SIMMEL Georg (1992), Le Conflit, Saulxures, Circé.

SIMMEL Georg (1999), Sociologie. Etudes sur les formes de la socialisation, Paris, PUF.

STOETZEL Jean (1943), Théorie des opinions, Paris, PUF.

TOUSSAINT Hergard (1958), « La procédure de conciliation en République fédérale d'Allemagne, en Suisse, en Autriche, en Scandinavie, en Angleterre et aux Etats-Unis d'Amérique », in Bulletin international des sciences sociales, « Techniques de médiation et de conciliation », volume X, n 4 , Unesco, p. 654663. 